\title{
CLOSURE RELATIONS FOR TOTALLY NONNEGATIVE CELLS IN
}

$G / P$.

\section{K. RIETSCH}

\begin{abstract}
The totally nonnegative part of a partial flag variety $G / P$ has been shown in $[11,10]$ to be a union of algbraic cells. We show that the closure of a cell is a union of cells and give a combinatorial description of the closure relations. The totally nonnegative cells are defined by intersecting $(G / P)_{\geq 0}$ with a certain stratification of $G / P$ defined by Lusztig [7]. We also verify the same closure relations for these strata.
\end{abstract}

\section{Introduction}

For a reductive algebraic group over $\mathbb{C}$ split over $\mathbb{R}$ with fixed choice of Chevalley generators in the Lie algebra, there is a well defined notion of positive, or $\mathbb{R}_{>0}$-valued, points due to Lusztig [5]. In the case of $G L_{n}$ with the standard choices the resulting " $G L_{n}\left(\mathbb{R}_{>0}\right)$ " recovers the classical notion of totally positive matrices, that is matrices all of whose minors are in $\mathbb{R}_{>0}$. For general $G$ the set $G\left(\mathbb{R}_{>0}\right)$, or $G_{>0}$ as we will denote it, is therefore called the totally positive part of $G$. The closure $G_{\geq 0}$ of $G_{>0}$ (in the real topology) is called the totally nonnegative part of $G$.

These notions extend in a natural way to flag varieties $G / P,[5,6]$. That is, one has a notion of $(G / P)_{>0}$ - this is a semi-algebraic subset of the real points in $G / P$ - and of $(G / P)_{\geq 0}$, the closure of $(G / P)_{>0}$. Now recall that $G / B$ has a decomposition into smooth strata $\mathcal{R}_{v, w}$, obtained as intersections of opposed Bruhat cells and indexed by pairs $v, w$ in the Weyl group with $v \leq w$. In [7] Lusztig defined an analogous decomposition of $G / P$ into smooth strata $\mathcal{P}_{x, u, w}$. These decompositions intersected with the $(G / P)_{\geq 0}$ give cell decompositions of the totally nonnegative parts of the $G / P,[11,10]$. We call the components of this decomposition of $(G / P)_{\geq 0}$ the totally nonnegative cells in $G / P$. Note that there is one open totally nonnegative cell in $G / P$, namely $(G / P)_{>0}$ itself.

It was proved in $[6]$ that $(G / B)_{\geq 0}$ is contractible, and the same holds for $(G / P)_{\geq 0}$ by the same proof. Also Fomin and Shapiro [1] studied links of totally nonnegative cells inside a big cell of $S L_{n} / B$, in particular showing them to be contractible. Beyond these special cases, however, not much is known about the closures of the individual cells or how the cells are glued together.

In this paper we prove that the closure of a totally nonnegative cell is a union of totally nonnegative cells and describe the closure relations in terms of the Weyl group. In the full flag variety case we show that $\mathcal{R}_{v^{\prime}, w^{\prime}}^{>0} \subseteq \overline{\mathcal{R}_{v, w}^{>0}}$, whenever $v \leq v^{\prime} \leq w^{\prime} \leq w$,

Received by the editors September 15, 2005.

2000 Mathematics Subject Classification. 14m15; $20 \mathrm{G} 15$.

Key words and phrases. Algebraic groups, partial flag varieties, total positivity.

The author is supported by a Royal Society Dorothy Hodgkin Research Fellowship. 


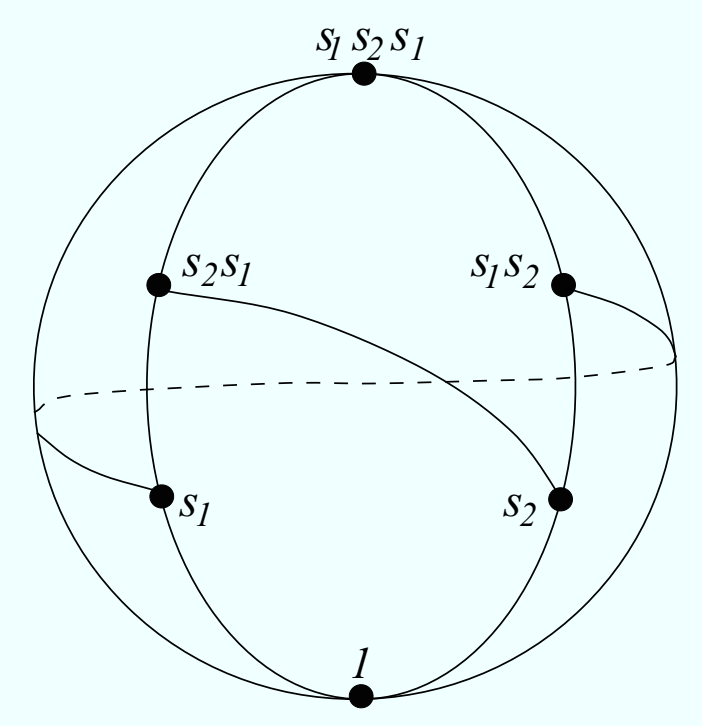

FiguRE 1. $\left(S L_{3} / B\right)_{\geq 0}$ with its cell decomposition

see Theorem 4.1. This theorem is then generalized to $G / P$ (see Theorem 6.1), with the combinatorial description of the cells and their closure relations given in Section 6 .

A main difficulty in proving such results lies in how to find a totally nonnegative cell $\mathcal{R}_{v, w}^{>0}$ inside the closure of another. While much detailed information is available about the individual cells, such as parameterizations, explicit defining equalities/inequalities (see [9]), none of these results readily extend to the closures of the cells. The central idea which allows us to relate this problem to an easier special case is contained in Lemma 4.3.

The combinatorial properties of our poset describing the closure relations between totally nonnegative cells were recently investigated by Williams [14]. Her results suggest, and she conjectures, that $(G / P)_{\geq 0}$ is a regular CW complex homeomorphic to a closed ball. For an illustration of the totally nonnegative part with its cell decomposition in the case of $S L_{3} / B$ see Figure 1.

In the last section we verify the same closure relations as among the totally nonnegative cells, for the strata $\mathcal{P}_{x, u, w}$ of $G / P$. In this case $G / P$ is either taken again over $\mathbb{R}$, or over an algebraically closed field $\mathbb{K}$ (and Zariski topology). The closure relations among the totally nonnegative cells could in retrospect be viewed as an $\mathbb{R}_{>0^{-}}$ valued analogue of Proposition 7.2, although the results involving positivity are more difficult to prove.

Lusztig's stratification of $G / P$ (over $\mathbb{C}$ ) has recently been reinterpreted by Goodearl and Yakimov [2] in a Poisson geometric setting. Namely the strata arise as torusorbits of symplectic leaves for a certain natural Poisson structure on $G / P$. Their paper also independently gives closure relations among these strata [2, Theorem 1.8] which look quite different from our Proposition 7.2. The combinatorial equivalence 
of their description of the poset structure with ours was recently proved by Xuhua He [13] using arguments from [3].

Acknowledgements. I am grateful to Lauren Williams for prompting me to think about the closure relations between the totally nonnegative cells and for useful discussions.

\section{Preliminaries}

2.1. We recall some basic notation and results from algebraic groups, see e.g. [12]. Suppose $\mathbb{K}$ is an algebraically closed field, $\mathbb{K}=\overline{\mathbb{K}}$, or $\mathbb{K}=\mathbb{R}$. Let $G$ be a semisimple linear algebraic group over $\overline{\mathbb{K}}$ split over $\mathbb{K}$. We identify $G$ and any related spaces with their $\mathbb{K}$-valued points. If $\mathbb{K}=\mathbb{R}$ we consider them with their real topology (as real manifolds or subsets thereof), otherwise we consider their Zariski topology.

Let $T$ be a split torus and $B^{+}$and $B^{-}$opposite Borel subgroups containing $T$. The unipotent radicals of $B^{+}$and $B^{-}$are denoted $U^{+}$and $U^{-}$, respectively. Let $\left\{\alpha_{i} \mid i \in I\right\}$ be the set of simple roots associated to $B^{+}$and $\left\{\alpha_{i}^{\vee} \mid i \in I\right\}$ the corresponding coroots. Then we have the simple root subgroups $U_{\alpha_{i}}^{+} \subseteq U^{+}$and $U_{\alpha_{i}}^{-} \subseteq U^{-}$. Furthermore assume we are given homomorphisms

$$
\phi_{i}: S L_{2}(\mathbb{K}) \rightarrow G, \quad i \in I,
$$

such that

and such that

$$
\phi_{i}\left(\left(\begin{array}{cc}
t & 0 \\
0 & t^{-1}
\end{array}\right)\right)=\alpha_{i}^{\vee}(t), \quad t \in \mathbb{K}^{*}
$$

$$
\phi_{i}\left(\left(\begin{array}{cc}
1 & m \\
0 & 1
\end{array}\right)\right):=x_{i}(m), \quad \phi_{i}\left(\left(\begin{array}{cc}
1 & 0 \\
m & 1
\end{array}\right)\right):=y_{i}(m),
$$

define isomorphisms $x_{i}: \mathbb{K} \rightarrow U_{\alpha_{i}}^{+}$and $y_{i}: \mathbb{K} \rightarrow U_{\alpha_{i}}^{-}$.

Let $W=N_{G}(T) / T$ be the Weyl group of $G$. For $i \in I$ the elements

$$
\dot{s}_{i}=x_{i}(-1) y_{i}(1) x_{i}(-1)
$$

represent the simple reflections $s_{i} \in W$. If $w=s_{i_{1}} \ldots s_{i_{m}}$ is a reduced expression for $w$ then we write $\ell(w)=m$ for the length of $w$. It is also known that the representative

$$
\dot{w}=\dot{s}_{i_{1}} \ldots \dot{s}_{i_{m}}
$$

of $w$ is well defined, independent of the choice of reduced expression. Inside $W$ we have a longest element which is denoted by $w_{0}$.

2.2. Let $J \subseteq I$. The parabolic subgroup $W_{J} \subseteq W$ is the subgroup generated by all of the $s_{j}$ with $j \in J$. Let $w_{J}$ denote the longest element in $W_{J}$. We also consider the set $W^{J}$ of minimal coset representatives for $W / W_{J}$, and the set $W_{\max }^{J}=W^{J} w_{J}$ of maximal coset representatives.

The parabolic subgroup $W_{J}$ of $W$ corresponds to a parabolic subgroup $P_{J}$ in $G$ containing $B^{+}$. Namely, $P_{J}$ is the subgroup of $G$ generated by $B^{+}$and the elements $\dot{w}$ for $w \in W_{J}$. Let $\mathcal{P}^{J}$ be the set of parabolic subgroups $P$ conjugate to $P_{J}$. This is a homogeneous space for the conjugation action of $G$ and can be identified with the partial flag variety $G / P_{J}$ via

$$
G / P_{J} \stackrel{\sim}{\longrightarrow} \mathcal{P}^{J}: g P_{J} \mapsto g P_{J} g^{-1} .
$$


In the case $J=\emptyset$ we are identifying the full flag variety $G / B^{+}$with the variety $\mathcal{B}$ of Borel subgroups in $G$. We have the usual projection from the full flag variety to any partial flag variety which takes the form $\pi=\pi^{J}: \mathcal{B} \rightarrow \mathcal{P}^{J}$, where $\pi(B)$ is the unique parabolic subgroup of type $J$ containing $B$.

The conjugate of a parabolic subgroup $P$ by an element $g \in G$ will be denoted by $g \cdot P:=g P g^{-1}$.

2.3. Recall the Bruhat decomposition for the full flag variety,

$$
\mathcal{B}=\bigsqcup_{w \in W} B^{+} \dot{w} \cdot B^{+},
$$

and the Bruhat order $\leq$ on $W$. The Bruhat cell $B^{+} \dot{w} \cdot B^{+}$is isomorphic to $\mathbb{K}^{\ell(w)}$. And the Bruhat order has the property

$$
v \leq w \quad \Longleftrightarrow \quad B^{+} \dot{v} \cdot B^{+} \subseteq \overline{B^{+} \dot{w} \cdot B^{+}},
$$

for $v, w \in W$.

It is a well known consequence of Bruhat decomposition that $\mathcal{B} \times \mathcal{B}$ is the union of the $G$-orbits $\mathcal{O}(w)=G \cdot\left(B^{+}, \dot{w} \cdot B^{+}\right)$, with $G$ acting diagonally. Therefore to any pair $\left(B_{1}, B_{2}\right)$ of Borel subgroups one can associate a unique $w \in W$ such that

$$
\left(B_{1}, B_{2}\right)=\left(g \cdot B^{+}, g \dot{w} \cdot B^{+}\right)
$$

for some $g \in G$. We write

$$
B_{1} \stackrel{w}{\rightarrow} B_{2}
$$

in this case and call $w$ the relative position of $B_{1}$ and $B_{2}$.

2.4. Finally, let us consider the two opposite Bruhat decompositions

$$
\mathcal{B}=\bigsqcup_{w \in W} B^{+} \dot{w} \cdot B^{+}=\bigsqcup_{v \in W} B^{-} \dot{v} \cdot B^{+} .
$$

Note that $B^{-} \dot{v} \cdot B^{+} \cong \mathbb{K}^{\ell\left(w_{0}\right)-\ell(v)}$. The closure relations for these opposite Bruhat cells are given by $B^{-} \dot{v}^{\prime} \cdot B^{+} \subset \overline{B^{-} \dot{v} \cdot B^{+}}$if and only if $v \leq v^{\prime}$. We define

$$
\mathcal{R}_{v, w}:=B^{+} \dot{w} \cdot B^{+} \cap B^{-} \dot{v} \cdot B^{+},
$$

the intersection of opposed Bruhat cells. This intersection is empty unless $v \leq w$, in which case it is smooth of dimension $\ell(w)-\ell(v)$, see $[4,7]$.

\section{Total Positivity for $G$ and $\mathcal{B}$}

Let $\mathbb{K}=\mathbb{R}$. The totally nonnegative part $G_{\geq 0}$ of $G$ is defined by Lusztig [5] to be the semigroup inside $G$ generated by the sets

$$
\begin{aligned}
& \left\{x_{i}(t) \mid t \in \mathbb{R}_{>0}, i \in I\right\}, \\
& \left\{y_{i}(t) \mid t \in \mathbb{R}_{>0}, i \in I\right\}, \text { and } \\
& T_{>0}:=\left\{t \in T \mid \chi(t)>0 \text { all } \chi \in X^{*}(T)\right\} .
\end{aligned}
$$

When $G=S L_{n}(\mathbb{R})$ then by a Theorem of A. Whitney's this definition agrees with the classical notion of totally nonnegative matrices inside $S L_{n}(\mathbb{R})$, that is those matrices all of whose minors are nonnegative. 
3.1. We recall some basic facts about total positivity for $G$ from [5]. Let $U_{\geq 0}^{+}:=$ $G_{\geq 0} \cap U^{+}$and $U_{\geq 0}^{-}:=G_{\geq 0} \cap U^{-}$. For $w \in W$ and $s_{i_{1}} \ldots s_{i_{m}}=w$ a reduced expression define

$$
\begin{aligned}
U^{+}(w) & :=\left\{x_{i_{1}}\left(t_{1}\right) x_{i_{2}}\left(t_{2}\right) \ldots x_{i_{m}}\left(t_{m}\right) \mid t_{i} \in \mathbb{R}_{>0}\right\} \\
U^{-}(w) & :=\left\{y_{i_{1}}\left(t_{1}\right) y_{i_{2}}\left(t_{2}\right) \ldots y_{i_{m}}\left(t_{m}\right) \mid t_{i} \in \mathbb{R}_{>0}\right\}
\end{aligned}
$$

These sets are independent of the chosen reduced expression and give

$$
\begin{aligned}
& U^{+}(w)=U_{\geq 0}^{+} \cap B^{-} \dot{w} B^{-}, \\
& U^{-}(w)=U_{\geq 0}^{-} \cap B^{+} \dot{w} B^{+} .
\end{aligned}
$$

In particular $U_{\geq 0}^{+}=\bigsqcup_{w \in W} U^{+}(w)$ and $U_{\geq 0}^{-}=\bigsqcup_{w \in W} U^{-}(w)$. Moreover $U^{+}(w)$ and $U^{-}(w)$ are isomorphic to $\mathbb{R}_{>0}^{\ell(w)}$ using the $t_{i}$ as coordinates.

Suppose $v \leq w$ then $U^{+}(v)$ can be obtained from $U^{+}(w)$ by letting certain of the $t_{i}$ coordinates tend to zero. So $U^{+}(v)$ lies in the closure of $U^{+}(w)$. Moreover the condition $v \leq w$ is necessary by the analogous property of the Bruhat decomposition. The same goes for the $U^{-}(w)$. Since $U_{\geq 0}^{+}$and $U_{\geq 0}^{-}$are closed in $G$ by [5, Proposition 4.2], we have

$$
\overline{U^{+}(w)}=\bigsqcup_{v \leq w} U^{+}(v), \quad \overline{U^{-}(w)}=\bigsqcup_{v \leq w} U^{-}(w) .
$$

Note that in particular $\overline{U^{+}\left(w_{0}\right)}=U_{\geq 0}^{+}$and $\overline{U^{-}\left(w_{0}\right)}=U_{\geq 0}^{-}$. The totally positive parts for $U^{+}$and $U^{-}$are defined by

$$
U_{>0}^{+}:=U^{+}\left(w_{0}\right), \quad U_{>0}^{-}:=U^{-}\left(w_{0}\right) .
$$

3.2. The totally positive and totally nonnegative parts of the flag variety $\mathcal{B}$ are defined by

$$
\begin{aligned}
& \mathcal{B}_{>0}:=\left\{y \cdot B^{+} \mid y \in U_{>0}^{-}\right\}, \\
& \mathcal{B}_{\geq 0}:=\overline{\mathcal{B}_{>0}} .
\end{aligned}
$$

By $\left[5\right.$, Theorem 8.7] $\mathcal{B}_{>0}$ can be described in a symmetric way as

$$
\mathcal{B}_{>0}=\left\{x \cdot B^{-} \mid x \in U_{>0}^{+}\right\} .
$$

In other words $\mathcal{B}_{>0}$ is invariant under the automorphism of $G$ (and hence $\mathcal{B}$ ) which swaps the $x_{i}(t)$ and the $y_{i}(t)$.

3.3. The set $\mathcal{B}_{\geq 0}$ again has a cell decomposition, which was conjectured in [5] and proved in [11]. This result was also proved again in [9] and with explicit descriptions of the cells given. We recall the construction from [9] below.

Let $v \leq w$ and let $\mathbf{w}=\left(i_{1}, \ldots, i_{m}\right)$ encode a reduced expression $s_{i_{1}} \ldots s_{i_{m}}$ for $w$. Then there exists a unique subexpression $s_{i_{j_{1}}} \ldots s_{i_{j_{k}}}$ for $v$ in $\mathbf{w}$ with the property that, for $l=1, \ldots, k$,

$$
s_{i_{j_{1}}} \ldots s_{i_{j_{l}}} s_{i_{r}}>s_{i_{j_{1}}} \ldots s_{i_{j_{l}}} \quad \text { whenever } j_{l}<r \leq j_{l+1},
$$

where $j_{k+1}:=m$. It is the rightmost reduced subexpression for $v$ in $\mathbf{w}$ and we denote it by $\mathbf{v}=\left(j_{1}, \ldots, j_{k}\right)$. 
Then we define

$$
\mathcal{R}_{v, w}^{>0}:=\left\{g_{1} \ldots g_{m} \cdot B^{+} \mid \text {where } g_{r}=\left\{\begin{array}{ll}
\dot{s}_{i_{r}}, & \text { if } r \in\left\{j_{1}, \ldots, j_{k}\right\}, \\
y_{i_{r}}\left(t_{r}\right), t_{r} \in \mathbb{R}_{>0} & \text { otherwise. }
\end{array}\right\}\right.
$$

By [9, Theorem 11.3] we have that this definition is independent of the reduced expression for $w$, and

$$
\mathcal{R}_{v, w}^{>0}=\mathcal{R}_{v, w} \cap \mathcal{B}_{\geq 0} .
$$

Moreover the $\mathcal{R}_{v, w}^{>0}$ are isomorphic to $\mathbb{R}_{>0}^{\ell(w)-\ell(v)}$. So this gives an explicit decomposition of $\mathcal{B}_{\geq 0}$ into cells.

We also write $\left(B^{+} \dot{w} \cdot B^{+}\right)_{\geq 0}$ for the intersection of the Bruhat cell with $\mathcal{B}_{\geq 0}$. Then of course

$$
\left(B^{+} \dot{w} \cdot B^{+}\right)_{\geq 0}=\bigsqcup_{v ; v \leq w} \mathcal{R}_{v, w}^{>0} .
$$

If $v=1$ then we have $\mathcal{R}_{1, w}=U^{-}(w) \cdot B^{+}$, and the above decomposition of $\mathcal{B}_{\geq 0}$ extends Lusztig's cell decomposition of $U_{\geq 0}^{-} \cong U_{\geq 0}^{-} \cdot B^{+}$.

3.4. We can apply the symmetry from Section 3.2 to get an alternate description for the $\mathcal{R}_{v, w}^{>0}$. Namely let

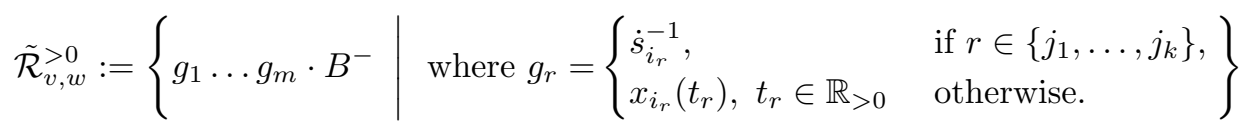

with the same notation as in Section 3.3. Then it follows that

$$
\tilde{\mathcal{R}}_{v, w}^{>0}=\left(B^{+} \dot{v} \cdot B^{-} \cap B^{-} \dot{w} \cdot B^{-}\right) \cap \mathcal{B}_{\geq 0}=\mathcal{R}_{w w_{0}, v w_{0}}^{>0} .
$$

3.5. Suppose $w, w_{1}, w_{2} \in W$ with $w=w_{1} w_{2}$ and such that the lengths add, $\ell(w)=$ $\ell\left(w_{1}\right)+\ell\left(w_{2}\right)$. Then there is a well defined map

$$
\begin{aligned}
\pi_{w_{1}}^{w}: B^{+} \dot{w} \cdot B^{+} & \rightarrow B^{+} \dot{w}_{1} \cdot B^{+} \\
B=z \dot{w} \cdot B^{+} & \mapsto z \dot{w}_{1} \cdot B^{+}
\end{aligned}
$$

where $z \in U^{+}$. We call $\pi_{w_{1}}^{w}$ a reduction map. The element $\pi_{w_{1}}^{w}(B)$ is uniquely determined by the property

$$
B^{+} \stackrel{w_{1}}{\longrightarrow} \pi_{w_{1}}^{w}(B) \stackrel{w_{2}}{\longrightarrow} B
$$

It was proved in [11] that $\pi_{w_{1}}^{w}$ preserves total positivity. That is, if $B=z \dot{w} \cdot B^{+} \in \mathcal{B}_{\geq 0}$ then $\pi_{w_{1}}^{w}(B)$ lies in $\mathcal{B}_{\geq 0}$.

Now let $B=g_{1} \ldots g_{m} \cdot B^{+} \in \mathcal{R}_{v, w}^{>0}$ where the factors $g_{i}$ and all the notation are as in Section 3.3. And additionally suppose the reduced expression for $w$ is a product of reduced expressions for $w_{1}$ and $w_{2}$, so $w_{1}=s_{i_{1}} \ldots s_{i_{m^{\prime}}}$ and $w_{2}=s_{i_{m^{\prime}+1}} \ldots s_{i_{m}}$. Then we have explicitly

$$
\pi_{w_{1}}^{w}\left(g_{1} g_{2} \ldots g_{m} \cdot B^{+}\right)=g_{1} \ldots g_{m^{\prime}} \cdot B^{+} .
$$

In particular the reduction map restricts to a map $\pi_{w_{1}}^{w}: \mathcal{R}_{v, w}^{>0} \rightarrow \mathcal{R}_{v_{\left(m^{\prime}\right)}, w_{1}}^{>0}$ where $v_{\left(m^{\prime}\right)}=s_{i_{j_{1}}} \ldots s_{i_{j_{p}}}$ for $j_{p} \leq m^{\prime}<j_{p+1}$. 


\section{Closure relations for the cells in $\mathcal{B}_{\geq 0}$}

The aim of this section is to prove the following theorem.

Theorem 4.1. Let $v, w \in W$. Then

$$
\overline{\mathcal{R}}>0, w=\bigsqcup_{v \leq v^{\prime} \leq w^{\prime} \leq w} \mathcal{R}_{v^{\prime}, w^{\prime}}^{>0}
$$

We begin with an easy special case.

Lemma 4.2. For $v \in W$,

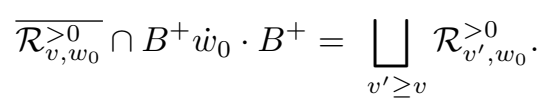

Proof. By Section 3.4 we can rewrite

$$
\mathcal{R}_{v^{\prime}, w_{0}}^{>0}=\tilde{\mathcal{R}}_{1, v^{\prime} w_{0}}^{>0}=U^{+}\left(v^{\prime} w_{0}\right) \dot{w}_{0} \cdot B^{+} .
$$

Now $v^{\prime} \geq v$ implies $v^{\prime} w_{0}^{-1} \leq v w_{0}$. Hence by Section 3.1 we have the inclusion $U^{+}\left(v^{\prime} w_{0}\right) \subseteq \overline{U^{+}\left(v w_{0}\right)}$, and therefore $\mathcal{R}_{v^{\prime}, w_{0}}^{>0} \subseteq \overline{\mathcal{R}_{v, w_{0}}^{>0}}$. The opposite inclusion follows from the closure relations of Bruhat decomposition.

Lemma 4.3. There is a homeomorphism

$$
\phi=\phi_{w}: U^{-}\left(w_{0} w^{-1}\right) \times\left(B^{+} \dot{w} \cdot B^{+}\right) \geq 0 \stackrel{\sim}{\longrightarrow} \bigsqcup_{v \leq w} \mathcal{R}_{v, w_{0}}^{>0}
$$

defined by $\phi(u, B):=u \cdot B$. Moreover

$$
\phi_{w}\left(U^{-}\left(w_{0} w^{-1}\right) \times \mathcal{R}_{v, w}^{>0}\right)=\mathcal{R}_{v, w_{0}}^{>0} .
$$

Proof. Choose a reduced expression $\mathbf{w}_{0}=\left(i_{1}, \ldots, i_{N}\right)$ for $w_{0}$ such that $\left(i_{1}, \ldots i_{r}\right)$ is a reduced expression for $w_{0} w^{-1}$. Then using the parameterizations of $U^{-}\left(w_{0} w^{-1}\right)$ and $\mathcal{R}_{v, w_{0}}^{>0}$ described in Sections 3.1 and 3.3, respectively, we see that

$$
\begin{aligned}
U^{-}\left(w_{0} w^{-1}\right) \times \mathcal{R}_{v, w}^{>0} & \stackrel{\sim}{\rightarrow} \mathcal{R}_{v, w_{0}}^{>0}, \\
(u, B) & \mapsto u \cdot B .
\end{aligned}
$$

is an isomorphism. Explicitly we have

$$
\mathcal{R}_{v, w_{0}}=\left\{y_{i_{1}}\left(t_{1}\right) \ldots y_{i_{r}}\left(t_{r}\right) g_{r+1} \ldots g_{N} \cdot B^{+} \mid g_{r+1} \ldots g_{N} \cdot B^{+} \in \mathcal{R}_{v, w}^{>0}\right\}
$$

Now applying the reduction map $\pi_{w_{0} w^{-1}}^{w_{0}}$ from Section 3.5 we get

$$
\begin{aligned}
\mathcal{R}_{v, w_{0}}^{>0} & \longrightarrow \mathcal{R}_{1, w_{0} w^{-1}}^{>0} \\
y_{i_{1}}\left(t_{1}\right) \ldots y_{i_{r}}\left(t_{r}\right) g_{r+1} \ldots g_{N} \cdot B^{+} & \mapsto y_{i_{1}}\left(t_{1}\right) \ldots y_{i_{r}}\left(t_{r}\right) \cdot B^{+}
\end{aligned}
$$

Note that $\pi_{w_{0} w^{-1}}^{w_{0}}$ is defined on the whole Bruhat cell $B^{+} \dot{w}_{0} \cdot B^{+}$. We can therefore combine these maps for varying $v$ and compose with the isomorphism $U^{-} \cdot B^{+} \stackrel{\sim}{\longrightarrow} U^{-}$, to get

$$
p_{1}: \bigsqcup_{v \leq w} \mathcal{R}_{v, w_{0}}^{>0} \rightarrow U^{-}\left(w_{0} w^{-1}\right)
$$


The inverse to $\phi$ is now given by

$$
\begin{aligned}
\psi: \bigsqcup_{v \leq w} \mathcal{R}_{v, w_{0}}^{>0} & \rightarrow U^{-}\left(w_{0} w^{-1}\right) \times\left(B^{+} \dot{w} \cdot B^{+}\right) \geq 0 \\
B & \mapsto\left(p_{1}(B), p_{1}(B)^{-1} \cdot B\right) .
\end{aligned}
$$

Lemma 4.4. Let $v \leq v^{\prime} \leq w \in W$. Then

$$
\mathcal{R}_{v^{\prime}, w}^{>0} \subseteq \overline{\mathcal{R}_{v, w}^{>0}}
$$

Proof. Using Lemma 4.2 we see that

$$
\mathcal{R}_{v^{\prime}, w_{0}}^{>0} \subset \overline{\mathcal{R}_{v, w_{0}}^{>0}} \cap \bigsqcup_{x \leq w} \mathcal{R}_{x, w_{0}}^{>0} .
$$

Applying $\psi:=\phi^{-1}$ from Lemma 4.3 to both sides of this inclusion gives

$$
\psi\left(\mathcal{R}_{v^{\prime}, w_{0}}^{>0}\right) \subset \psi\left(\overline{\mathcal{R}_{v, w_{0}}^{>0}} \cap \bigsqcup_{x \leq w} \mathcal{R}_{x, w_{0}}^{>0}\right) \subset \overline{\psi\left(\mathcal{R}_{v, w_{0}}^{>0}\right)} .
$$

Therefore

$$
U^{-}\left(w_{0} w^{-1}\right) \times \mathcal{R}_{v^{\prime}, w}^{>0}=\psi\left(\mathcal{R}_{v^{\prime}, w_{0}}^{>0}\right) \subset \overline{\psi\left(\mathcal{R}_{v, w_{0}}^{>0}\right)}=\overline{U^{-}\left(w_{0} w^{-1}\right) \times \mathcal{R}_{v, w}^{>0}},
$$

where we may take the closure on the right hand side to be the closure inside the domain of $\phi$, that is inside $U^{-}\left(w_{0} w^{-1}\right) \times\left(B^{+} \dot{w} \cdot B^{+}\right)_{\geq 0}$. It follows that $\mathcal{R}_{v^{\prime}, w}^{>0} \subseteq$ $\overline{\mathcal{R}>0}$.

Proof of Theorem 4.1. By [5] $\mathcal{B}_{\geq 0}$ is symmetric with respect to interchanging $B^{+}$ and $B^{-}$, see Section 3.2. Therefore it follows that Lemma 4.4 also holds for the $\tilde{\mathcal{R}}_{v, w}^{>0}$ defined in Section 3.4. That is, $\tilde{\mathcal{R}}_{v^{\prime}, w}^{>0} \subseteq \overline{\tilde{\mathcal{R}}_{v, w}^{>0}}$ whenever $v \leq v^{\prime} \leq w$. Now if $v \leq v^{\prime} \leq w^{\prime} \leq w$, then

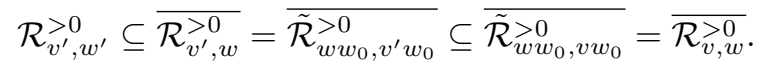

This shows the inclusion $\supseteq$ in the statement of Theorem 4.1.

The other inclusion is clear from the closure relations of Bruhat decomposition. $\mathcal{R}_{v^{\prime}, w^{\prime}}^{>0} \cap \overline{\mathcal{R}_{v, w}^{>0}} \neq \emptyset$ implies on the one hand $\mathcal{R}_{v^{\prime}, w^{\prime}}^{>0} \cap \overline{B^{-} \dot{v} \cdot B^{+}} \neq \emptyset$ and on the other hand $\mathcal{R}_{v^{\prime}, w^{\prime}}^{>0} \cap \overline{B^{+} \dot{w} \cdot B^{+}} \neq \emptyset$. So we must have $v \leq v^{\prime}$ and $w^{\prime} \leq w$.

We note that Theorem 4.1 implies $\overline{\mathcal{R}_{v, w}} \cap \mathcal{B}_{\geq 0}=\overline{\mathcal{R}_{v, w}^{>0}}$.

\section{Lusztig's decomposition of $\mathcal{P}^{J}$}

The stratification of $\mathcal{B}$ into smooth pieces $\mathcal{R}_{v, w}$ has an analogue for partial flag varieties introduced by Lusztig in [7].

Consider a triple of Weyl group elements $x, u, w \in W$ with $x \in W_{\max }^{J}, w \in W^{J}$ and $u \in W_{J}$. Then $\mathcal{P}_{x, u, w}^{J} \subset \mathcal{P}^{J}$ is defined as the set of all $P \in \mathcal{P}^{J}$ such that there exist Borel subgroups $B_{L}$ and $B_{R}$ inside $P$ satisfying

$$
B^{+} \stackrel{w}{\longrightarrow} B_{L} \stackrel{u}{\longrightarrow} B_{R} \stackrel{x^{-1} w_{0}}{\longrightarrow} B^{-} .
$$


An equivalent characterization of $\mathcal{P}_{x, u, w}^{J}$ is

$$
\mathcal{P}_{x, u, w}^{J}=\pi^{J}\left(\mathcal{R}_{x, w u}\right)=\pi^{J}\left(\mathcal{R}_{x u^{-1}, w}\right)
$$

It is not hard to see that $B_{L}$ and $B_{R}$ are uniquely determined as the Borel subgroups in $P$ 'closest to' $B^{+}$respectively $B^{-}$with regard to their relative position, and the projection maps $\mathcal{R}_{x, w u} \rightarrow \mathcal{P}_{x, u, v}^{J}$ and $\mathcal{R}_{x u^{-1}, w} \rightarrow \mathcal{P}_{x, u, v}^{J}$ are isomorphisms. In particular $\mathcal{P}_{x, u, w}^{J}$ is nonempty if and only if $x \leq w u$, in which case it is smooth of dimension $\ell(w)+\ell(u)-\ell(x)$.

Let us denote the indexing set for this decomposition of $\mathcal{P}^{J}$ by $Q^{J}$. So

$$
Q^{J}:=\left\{(x, u, w) \in W_{\max }^{J} \times W_{J} \times W^{J} \mid x \leq w u\right\} .
$$

We define the following partial oder on $Q^{J}$.

Definition 5.1. Let $\left(x^{\prime}, u^{\prime}, w^{\prime}\right)$ and $(x, u, w)$ in $Q^{J}$. Then define

$$
\left(x^{\prime}, u^{\prime}, w^{\prime}\right) \leq(x, u, w)
$$

if and only if there exist $u_{1}^{\prime}, u_{2}^{\prime} \in W_{J}$ satisfying $u_{1}^{\prime} u_{2}^{\prime}=u^{\prime}$ with $\ell\left(u_{1}^{\prime}\right)+\ell\left(u_{2}^{\prime}\right)=\ell\left(u^{\prime}\right)$, and such that

$$
x u^{-1} \leq x^{\prime} u_{2}^{\prime-1} \leq w^{\prime} u_{1}^{\prime} \leq w
$$

\section{Totally nonnegative cells in $\mathcal{P}^{J}$ and their closure relations}

The totally positive and nonnegative parts of $\mathcal{P}^{J}$ are defined in [6] by

$$
\begin{aligned}
& \mathcal{P}_{>0}^{J}=\pi^{J}\left(\mathcal{B}_{>0}\right), \\
& \mathcal{P}_{\geq 0}^{J}=\pi^{J}\left(\mathcal{B}_{\geq 0}\right) .
\end{aligned}
$$

Since $\pi^{J}$ is closed it follows that $\mathcal{P}_{\geq 0}^{J}=\overline{\mathcal{P}_{>0}^{J}}$.

We decompose $\mathcal{P}_{>0}^{J}$ by intersecting it with the strata $\mathcal{P}_{x, u, w}^{J}$ from Section 5 . From the definitions and the fact that reduction preserves total positivity it follows that ([10, Lemma 3.2])

$$
\mathcal{P}_{x, u, w ;>0}^{J}:=\mathcal{P}_{x, u, w}^{J} \cap \mathcal{P}_{\geq 0}^{J}=\pi^{J}\left(\mathcal{R}_{x, w u}^{>0}\right)=\pi^{J}\left(\mathcal{R}_{x u^{-1}, w}^{>0}\right) .
$$

Keeping in mind that $\pi^{J}: \mathcal{R}_{x, w u} \rightarrow \mathcal{P}_{x, u, w}^{J}$, say, is an isomorphism, we see that

$$
\mathcal{P}_{x, u, w ;>0}^{J} \cong \mathcal{R}_{x, w u}^{>0} \cong \mathbb{R}_{>0}^{\ell(w)+\ell(u)-\ell(x)}
$$

for any triple $(x, u, w) \in Q^{J}$.

We will prove the following theorem describing the closure relations between the strata $\mathcal{P}_{x, u, w ;>0}^{J}$ in $\mathcal{P}_{\geq 0}^{J}$.

Theorem 6.1. Let $(x, u, w) \in Q^{J}$ with partial order as in Definition 5.1, then

$$
\overline{\mathcal{P}_{x, u, w ;>0}^{J}}=\bigsqcup_{\left(x^{\prime}, u^{\prime}, w^{\prime}\right) \leq(x, u, w)} \mathcal{P}_{x^{\prime}, u^{\prime}, w^{\prime} ;>0}^{J} .
$$


Proof. Note first that we have $\overline{\mathcal{P}_{x, u, w ;>0}^{J}}=\pi^{J}\left(\overline{\mathcal{R}_{x u^{-1}, w}^{>0}}\right)=\pi^{J}\left(\overline{\mathcal{R}_{x, w u}^{>0}}\right)$.

Now suppose $\left(x^{\prime}, u^{\prime}, w^{\prime}\right) \leq(x, u, w)$. So we have $u_{1}^{\prime}$ and $u_{2}^{\prime}$ as in Definition 5.1. Let $P^{\prime} \in \mathcal{P}_{x^{\prime}, u^{\prime}, w^{\prime}}^{>0}$ with its associated Borel subgroups $B_{L}^{\prime} \in \mathcal{R}_{x^{\prime} u^{\prime-1}, w^{\prime}}^{>0}$ and $B_{R}^{\prime} \in \mathcal{R}_{x^{\prime}, w^{\prime} u^{\prime}}^{>0}$ such that $B_{L}^{\prime} \subset P^{\prime}$ and $B_{R}^{\prime} \subset P^{\prime}$. In particular

$$
B^{+} \stackrel{w^{\prime}}{\longrightarrow} B_{L}^{\prime} \stackrel{u^{\prime}}{\longrightarrow} B_{R}^{\prime} \stackrel{x^{\prime-1} w_{0}}{\longrightarrow} B^{-} .
$$

Let $B^{\prime}:=\pi_{w^{\prime} u_{1}^{\prime}}^{w^{\prime}{ }^{\prime}}\left(B_{R}^{\prime}\right)$ using the reduction map from Section 3.5. Then we have

$$
B^{+} \stackrel{w^{\prime}}{\longrightarrow} B_{L}^{\prime} \stackrel{u_{1}^{\prime}}{\longrightarrow} B^{\prime} \stackrel{u_{2}^{\prime}}{\longrightarrow} B_{R}^{\prime} \stackrel{x^{\prime-1} w_{0}}{\longrightarrow} B^{-} .
$$

Since reduction preserves total positivity we have $B^{\prime} \in \mathcal{R}_{x^{\prime} u_{2}^{\prime-1}, w^{\prime} u_{1}^{\prime}}^{>0}$ Also $\pi^{J}\left(B_{L}^{\prime}\right)=$ $P^{\prime}$ and the fact that $\left(B_{L}^{\prime}, B^{\prime}\right)$ have relative position $u_{1}^{\prime} \in W_{J}$ implies that $\pi\left(B^{\prime}\right)=P^{\prime}$. Now by (5.1) together with Theorem 4.1 it follows that $\mathcal{R}_{x^{\prime} u_{2}^{\prime-1}, w^{\prime} u_{1}^{\prime}}^{>0} \subset \overline{\mathcal{R}_{x u^{-1}, w}^{>0}}$. Therefore $P^{\prime}=\pi\left(B^{\prime}\right) \in \pi\left(\overline{\mathcal{R}_{x u^{-1}, w}^{>0}}\right)=\overline{\mathcal{P}_{x, u, w ;>0}^{J}}$. This proves the inclusion $\supseteq$.

For the opposite inclusion suppose that $\mathcal{P}_{x^{\prime}, u^{\prime}, w^{\prime} ;>0} \cap \overline{\mathcal{P}_{x, u, w ;>0}} \neq \emptyset$. So let $P^{\prime}$ be an element of this intersection. Then there exists a $\tilde{B}^{\prime} \in \overline{\mathcal{R}_{x u^{-1}, w}^{>0}}$ such that $\pi\left(\tilde{B}^{\prime}\right)=P^{\prime}$. Since $\tilde{B}^{\prime} \subset P^{\prime}$ we have $u_{1}, u_{2} \in W_{J}$ such that

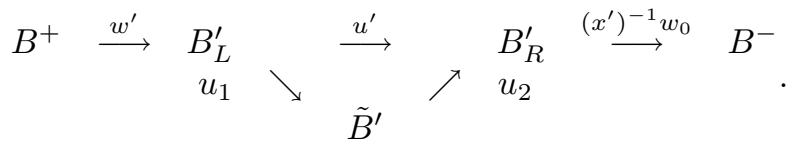

If it happens to be the case that $\ell\left(u_{1} u_{2}\right)=\ell\left(u_{1}\right)+\ell\left(u_{2}\right)$ then $u^{\prime}=u_{1} u_{2}$. So we can set $u_{1}^{\prime}=u_{1}$ and $u_{2}^{\prime}=u_{2}$ and are done. Otherwise there exists a simple reflection $s=s_{i_{1}} \in W_{J}$ such that $u_{1} s \leq u_{1}$ and $s u_{2} \leq u_{2}$. We have

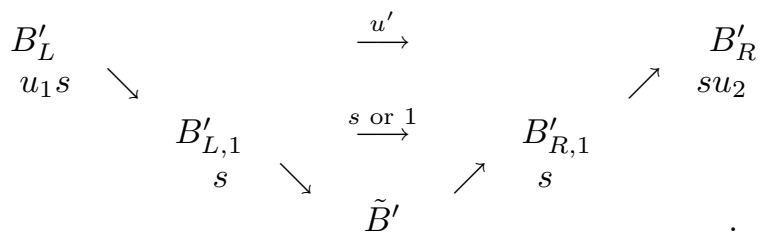

Here $B_{L, 1}^{\prime}$ is obtained from $\tilde{B}^{\prime}$ by reduction, $B_{L, 1}^{\prime}=\pi_{w u_{1} s}^{w u_{1}}\left(\tilde{B}^{\prime}\right)$. This implies $B_{L, 1}^{\prime} \in$ $\mathcal{B}_{\geq 0}$ (see Section 3.5). Using the inequalities

$$
x u^{-1} \leq x^{\prime} u_{2}^{-1} \leq x^{\prime}\left(s u_{2}\right)^{-1}, \quad w^{\prime} u_{1} s \leq w^{\prime} u_{1} \leq w
$$

and Theorem 4.1 it follows that

$$
B_{L, 1}^{\prime} \in \mathcal{R}_{x^{\prime} u_{2}^{-1}, w^{\prime} u_{1} s}^{>0} \sqcup \mathcal{R}_{x^{\prime}\left(s u_{2}\right)^{-1}, w^{\prime} u_{1} s}^{>0} \subseteq \overline{\mathcal{R}_{x u^{-1}, w}^{>0}} .
$$

Therefore we are now in the analogous situation as before

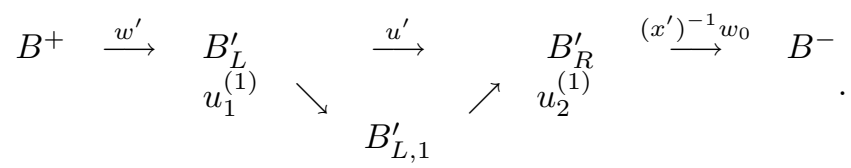

with totally nonnegative $B_{L, 1}^{\prime}$, but where $u_{1}^{(1)}=u_{1} s<u_{1}$. If again $\ell\left(u_{1}^{(1)} u_{2}^{(1)}\right) \neq$ $\ell\left(u_{1}^{(1)}\right)+\ell\left(u_{2}^{(1)}\right)$ then we can repeat the argument above. So we replace $u_{1}^{(1)}$ with a 
shorter $u_{1}^{(2)}=u_{1}^{(1)} s_{i_{2}}$, and $B_{L, 1}^{\prime}$ with $B_{L, 2}^{\prime}$ also in $\overline{\mathcal{R}_{x u^{-1}, w}^{>0}}$. Iterating this process we must eventually arrive at a case where $\ell\left(u_{1}^{(k)} u_{2}^{(k)}\right)=\ell\left(u_{1}^{(k)}\right)+\ell\left(u_{2}^{(k)}\right)$ (after at most $k=\ell\left(u_{1}\right)$ steps), at which point we set $u_{1}^{(k)}=u_{1}^{\prime}$ and $u_{2}^{(k)}=u_{2}^{\prime}$, and $B_{L, k}^{\prime} \in \overline{\mathcal{R}_{x u^{-1}, w}^{>0}}$ implies the inequalities (5.1).

\section{Closure relations for the strata $\mathcal{P}_{x, u, w}^{J}$ in $\mathcal{P}^{J}$}

Let $\mathbb{K}$ be again as in Section 2. Then we have a decomposition

$$
\mathcal{P}^{J}=\bigsqcup_{(x, u, w) \in Q^{J}} \mathcal{P}_{x, u, w}^{J}
$$

defined as in Section 6. We can now deduce the analogue of Theorem 6.1 for the strata $\mathcal{P}_{x, u, w}^{J}$ in $\mathcal{P}^{J}$.

Again the full flag variety case needs to be treated first. After that the proof proceeds in the same way as for Theorem 6.1.

Proposition 7.1. Let $v, w \in W$ with $v \leq w$. Then

$$
\overline{\mathcal{R}_{v, w}}=\bigsqcup_{v \leq v^{\prime} \leq w^{\prime} \leq w} \mathcal{R}_{v^{\prime}, w^{\prime}}
$$

The above result does not seem to appear in the literature, so we include a quick proof. It is however well-known to experts, [8].

Proof. The inclusion $\subseteq$ follows from the closure relations for Bruhat decomposition.

Let us prove the inclusion $\supseteq$. It suffices by $B^{+}-B^{-}$symmetry to show that $\mathcal{R}_{v^{\prime}, w} \subseteq$ $\overline{\mathcal{R}_{v, w}}$ whenever $v \leq v^{\prime}$ (as in the proof of Theorem 4.1). Consider the map

$$
\begin{aligned}
\gamma: B^{+} \dot{w} \cdot B^{+} \times\left(U^{-} \cap \dot{w} U^{-} \dot{w}^{-1}\right) & \longrightarrow \dot{w} U^{-} \cdot B^{+}, \\
(B, y) & \mapsto y \cdot B .
\end{aligned}
$$

It is an isomorphism and by restriction gives rise to isomorphisms

$$
\gamma_{v}: \mathcal{R}_{v, w} \times\left(U^{-} \cap \dot{w} U^{-} \dot{w}^{-1}\right) \longrightarrow B^{-} \dot{v} \cdot B^{+} \cap \dot{w} U^{-} \cdot B^{+},
$$

as in [6, Section 1.4]. Now if $v^{\prime} \geq v$ then we have

$$
B^{-} \dot{v}^{\prime} \cdot B^{+} \cap \dot{w} U^{-} \cdot B^{+} \subset \overline{B^{-} \dot{v} \cdot B^{+}} \cap \dot{w} U^{-} \cdot B^{+} .
$$

Applying $\gamma^{-1}$ to this inclusion we see that $\mathcal{R}_{v^{\prime}, w} \subset \overline{\mathcal{R}_{v, w}}$ and the proposition follows.

Now consider the partial flag variety case. Since $\pi^{J}$ is proper we have that $\overline{\mathcal{P}_{x, u, w}^{J}}=$ $\pi^{J}\left(\overline{\mathcal{R}_{x, w u}}\right)=\pi^{J}\left(\overline{\mathcal{R}_{x u^{-1}, w}}\right)$. The following proposition follows from the same proof as Theorem 6.1, only using Proposition 7.1 in place of Theorem 4.1 and leaving out the positivity considerations.

Proposition 7.2. Let $(x, u, w) \in Q^{J}$ with partial order as in Definition 5.1, then

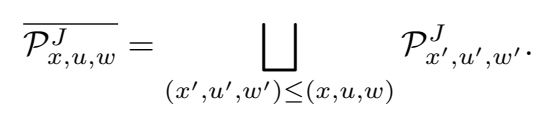




\section{References}

[1] S. Fomin and M. Shapiro, Stratified spaces formed by totally positive varieties, Michigan Math. J. (2000), 253-270.

[2] K. R. Goodearl and M. Yakimov, Poisson structures on affine spaces and flag varieties. II. General case, arXiv:math.QA/0509075.

[3] X. He, The G-stable pieces of the wonderful compactification, ArXiv Mathematics e-print arXiv:math/0412302 (2004), 22 pages.

[4] D. Kazhdan and G. Lusztig, Schubert varieties and Poincaré duality, Geometry of the Laplace operator (Proc. Sympos. Pure Math., Univ. Hawaii, Honolulu, Hawaii, 1979), Proc. Sympos. Pure Math., XXXVI, Amer. Math. Soc., Providence, R.I., 1980, pp. 185-203. MR 84g:14054

[5] George Lusztig, Total positivity in reductive groups, Lie theory and geometry: in honor of Bertram Kostant (G. I. Lehrer, ed.), Progress in Mathematics, vol. 123, Birkhaeuser, Boston, 1994, pp. 531-568.

[6] _ Introduction to total positivity, Positivity in Lie theory: open problems, de Gruyter Exp. Math., vol. 26, de Gruyter, Berlin, 1998, pp. 133-145. MR MR1648700 (99h:20077)

[7] _ , Total positivity in partial flag manifolds, Representation Theory 2 (1998), 70-78.

[8] , Personal communication, 2005.

[9] R. J. Marsh and K. Rietsch, Parametrizations in flag varieties, Representation Theory 8 (2004), $212-242$.

[10] K. Rietsch, Total positivity and real flag varieties, Ph.D. thesis, M.I.T., 1998.

[11] _ An algebraic cell decomposition of the nonnegative part of a flag variety, J. Algebra 213 (1999), 144-154.

[12] T. A. Springer, Linear algebraic groups, Second edition, Progress in Mathematics, vol. 9, Birkhäuser, Boston, 1998

[13] Lauren K. Williams, Personal communication.

[14] _ Shelling totally nonnegative flag varieties, arXiv:math.RT/0509129.

Department of Mathematics, King's College London, Strand, London WC2R 2LS

Department of Pure Mathematics, University of Waterloo, Canada

E-mail address: rietsch@mth.kcl.ac.uk 